\title{
MS17-P04 | EFFECT OF PRESSURE ON SLIT CHANNELS IN HYDRATE OF SODIUM SALT OF GUANINE: A LINK TO NUCLEOBASE INTERMOLECULAR INTERACTIONS
}

Gaydamaka, Anna (Novosibirsk State University, Novosibirsk, RUS); Arkhipov, Sergey (1Novosibirsk State University, Boreskov Institute of Catalysis SB RAS, Novosibirsk,, RUS); Zakharov, Boris (Novosibirsk State University, Boreskov Institute of Catalysis SB RAS, Novosibirsk, RUS); Seryotkin, Yuriy (Novosibirsk State University, V.S. Sobolev Institute of Geology and Mineralogy SB RAS, Novosibirsk, RUS); Boldyreva, Elena (Novosibirsk State University, Boreskov Institute of Catalysis SB RAS, Novosibirsk, RUS)

The studies of the biological and biomimetic objects are important not only for fundamental science but also for the design of drugs and materials.

The crystal structure of a hydrate of the sodium salt of guanine $\left(2 \mathrm{Na}^{+} \cdot \mathrm{C}_{5} \mathrm{H}_{3} \mathrm{~N}_{5} \mathrm{O}^{2-} \cdot 7 \mathrm{H}_{2} \mathrm{O}\right)$ [1] was studied using singlecrystal X-ray diffraction and Raman spectroscopy up to $\sim 2.5 \mathrm{GPa}$, after what the structure collapsed and the diffraction pattern disappeared. On increasing pressure, the distances between the double walls of the slit channels formed by the guanine moieties became more equal, the longer one shortening and the shorter one expanding. The topology of the $\mathrm{Na}$ - water intra-channel infinite clusters was preserved, although these clusters got distorted on compression. Among several types of the $\mathrm{NH}$... $\mathrm{O}$ and $\mathrm{OH}$...O hydrogen bonds, only two practically did not change with increasing pressure, namely the $\mathrm{OH} . . . \mathrm{O}$ and the $\mathrm{NH}$...O bonds connecting the guanine walls with the intra-channel water molecules. A possible relation to the interactions in the ion channels with the walls formed by the guanine species in biological systems is discussed.

Acknowledgements: The work was supported by the Russian Ministry of Science and High Education (project AAAA-A19-119020890025-3)

[1] D. Gur, L. Shimon, Acta Cryst. E, 2015, E71, pp. 282-283. 